\title{
Anti-reflux mucosal ablation (ARMA) as a new treatment for gastroesophageal reflux refractory to proton pump inhibitors: a pilot study
}

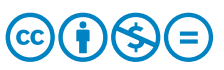

\begin{abstract}
Authors
Institutions

1 Digestive Diseases Center, Showa University Koto Toyosu Hospital, Tokyo, Japan

2 Department of Gastroenterology and Hepatology, Hospital Universitario Ramón y Cajal, Universidad de Alcalá, IRYCIS, Madrid, Spain
\end{abstract}

Haruhiro Inoue1, Mayo Tanabe1, Enrique Rodríguez de Santiago,2, Mary Raina Angeli Abad', Yuto Shimamura1, Yusuke Fujiyoshi ${ }^{1}$, Akiko Ueno ${ }^{1}$, Kazuya Sumi ${ }^{1}$, Hideomi Tomida ${ }^{1}$, Yugo Iwaya ${ }^{1}$, Haruo Ikeda ${ }^{1}$, Manabu Onimaru ${ }^{1}$

submitted 17.7.2019

accepted after revision 12.9 .2019

\author{
Bibliography \\ DOI https://doi.org/10.1055/a-1031-9436 | \\ Endoscopy International Open 2020; 08: E133-E138 \\ (c) Georg Thieme Verlag KG Stuttgart · New York \\ eISSN 2196-9736
}

Corresponding author

Mayo Tanabe, MD, Digestive Diseases Center, Showa University Koto Toyosu Hospital, 5-1-38, Toyosu, Koto-ku, Tokyo, 135-8577 Japan

Fax: +81-3-62046396

mayo.tanabe@gmail.com

\section{ABSTRACT}

Background The incidence of proton pump inhibitor (PPI)refractory gastroesophageal reflux disease (GERD) has been increasing. While surgical intervention with Laparoscopic Nissen Fundoplication remains the gold standard, less invasive anti-reflux interventions are desired. We have developed a minimally invasive anti-reflux mucosal ablation (ARMA) treatment. Herein, we report its technical details and describe its feasibility, safety, and efficacy in PPI-refractory GERD.

Methods We conducted a prospective single-center single-arm interventional trial evaluating the outcome of ARMA in 12 patients with PPI-refractory GERD. GERDHealth Related Quality of Life Questionnaire (GERD-HRQL) evaluation, Frequency Scale for the Symptoms of GERD (FSSG) assessment, and impedance-pH monitoring were performed at baseline and at 2 months post-ARMA.

Results A total of 12 patients underwent ARMA with a median follow-up duration of 9 months (range: 6 - 14 months). Median GERD-HRQL score significantly improved from 30.5 to $12(P=0.002)$; median FSSG score significantly improved from 25 to $10.5(P=0.002)$, and median DeMeester score decreased from 33.5 to $2.8(P=0.049)$ at 2 months followup. No immediate complications were observed.

Conclusion Our pilot study has shown that ARMA, a new endoscopic treatment for PPI-refractory GERD, is simple, safe, and improves GERD-related symptoms and objective acid reflux parameters.

\section{Introduction}

Gastroesophageal reflux disease (GERD) is a common disorder with an increasing prevalence [1]. Proton pump inhibitors (PPIs) are effective in achieving symptom control and preventing complications [2]. Approximately $40 \%$ of patients have persistent GERD symptoms despite PPI therapy, and there is increasing awareness with regard to the side effects of life-long

\footnotetext{
* The abstract of an earlier version of this article was presented at Digestive Disease Week 2019 (May 2019; San Diego, CA, USA).
}

PPI use [3,4]. While laparoscopic anti-reflux surgery (LARS) remains the gold standard for PPI-refractory GERD [5, 6], less invasive anti-reflux interventions are highly desired [7].

In 2014, we reported the first case series of anti-reflux mucosectomy (ARMS) as an endoscopic procedure to rebuild the flap valve at the gastric cardia [8]. In ARMS, scarring of the artificial ulcer created by endoscopic mucosal resection (EMR) or endoscopic submucosal dissection (ESD) at the gastric cardia tightens the enlarged cardiac opening. We encountered a post-ARMS patient with unresolved symptoms due to insufficient cardiac opening shrinkage ( $\triangleright$ Fig. 1$)$. However, additional 


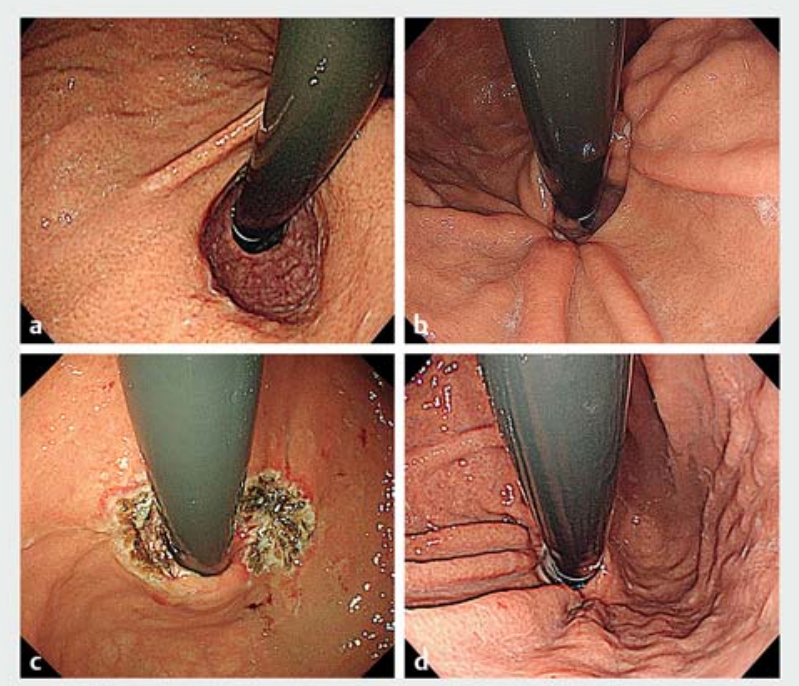

- Fig. 1 The first case of anti-reflux mucosal ablation (ARMA). a Pre-anti-reflux mucosectomy (ARMS). Endoscopy in retroflexion demonstrated significant valve opening/impairment (Flap valve grade III). b Post-ARMS. c Immediately post-ARMA. d Appearance at 1 month post-ARMA. Mucosal flap valve was re-shaped (Flap valve grade I).

ARMS was expected to be technically challenging due to scarring from the previous treatment. Thus, anti-reflux mucosal ablation (ARMA) was performed and the ablated area showed a shrinking effect similar to ARMS.

This study assesses the feasibility, efficacy, and safety of ARMA in PPI-refractory GERD.

\section{Materials and methods}

\section{Study design}

This prospective single-center single-arm interventional trial was conducted at Showa University Koto Toyosu Hospital during the period May 2018 - January 2019. Inclusion criteria were the presence of at least one typical reflux symptom more than two times a week despite double-dose PPI treatment for at least 6 months, and pathologic esophageal acid exposure defined by a DeMeester score $>14.7$ or acid exposure time (AET) $>4.2 \%$. Exclusion criteria were: age $<20$ years; primary esophageal motility disorders; sliding hiatal hernia $>3 \mathrm{~cm}$ in gastroscopy; grade IV Hill's flap valve, or pregnancy. High resolution esophageal manometry was performed in all patients to confirm the absence of esophageal motility disorders.

The primary outcome was symptom improvement evaluated by the GERD-Health Related Quality of Life Questionnaire (GERD-HRQL) [9] and the Frequency Scale for the Symptoms of GERD (FSSG) [10]. Secondary end points were improvement in DeMeester score and AET in 24-hour esophageal multichannel intraluminal impedance (MII)-pH monitoring, changes in cardia morphology assessed by Hill's flap grade [11], and safety.
This study was approved by Showa University Institutional review board (approval number: 17T5018) and registered on the University Hospital Medical Information Network (UMIN000032190). All participants provided written informed consent.

\section{Baseline assessment and follow-up}

All patients underwent gastroscopy, barium esophagography, high-resolution esophageal manometry, and MII-pH monitoring off PPI pre-ARMA. Baseline gastroscopy with esophageal biopsies was performed to rule out eosinophilic esophagitis. GERD-HRQL and FSSG questionnaires were completed by all patients pre- and 2 months post-ARMA. To compare the flap valve grade, grades I, II and III received scores of 0,1 and 2 points, respectively.

Two months post-ARMA, all patients were scheduled for reevaluation using gastroscopy and MII-pH monitoring off PPI.

\section{Anti-reflux mucosal ablation}

All procedures were performed by a single operator using a gastroscope (outer diameter, 9.9 mm; GIFQ260]; Olympus, Tokyo, Japan) with a transparent hood as a distal attachment and a triangle-tip knife J (Olympus).

Patients were instructed to fast for 6 hours pre-ARMA, which was performed under general anesthesia or propofol-based sedation. Patients were in the supine or left lateral decubitus position. The stomach was insufflated with $\mathrm{CO}_{2}$ to visualize the cardia in retroflex view. Markings were placed using the triangle-tip knife J connected to an electrocautery generator (VIO300D; ERBE Electromedizin, Tübingen, Germany) in spray coagulation mode ( $50 \mathrm{~W}$, effect 2 ). Mucosal ablation was planned around the cardia on the gastric side in a butterfly shape with width of approximately 1.5 scope diameter, leaving two contralateral areas of normal cardia mucosa with approximately one scope diameter, to avoid stenosis ( $\mathbf{F i g . 2 b}$ ). Saline with indigo carmine dye was injected into the submucosal layer along the markings using a 25 -gauge needle. A submucosal cushion reduces thermal injury and the risk of perforation during ablation. Mucosal ablation was performed using the triangle-tip knife J in spray coagulation mode (50 W, effect 2). Adequate ablation depth was defined as reaching the submucosal layer, which could be confirmed by observation of the indigo carmine dye during ablation. All patients received post-procedural single-dose PPI for 1 month.

\section{Statistical analysis}

Medians and ranges were used for continuous variables and frequencies with percentages for categorical data. Bivariate analyses pre- and post-ARMA were performed using the Wilcoxon matched-pairs signed-ranks test. Two-sided $P$ values $<0.05$ were considered to be statistically significant. Statistical analysis was performed using JMP 14 (SAS Institute, Cary, North Carolina, United States). 

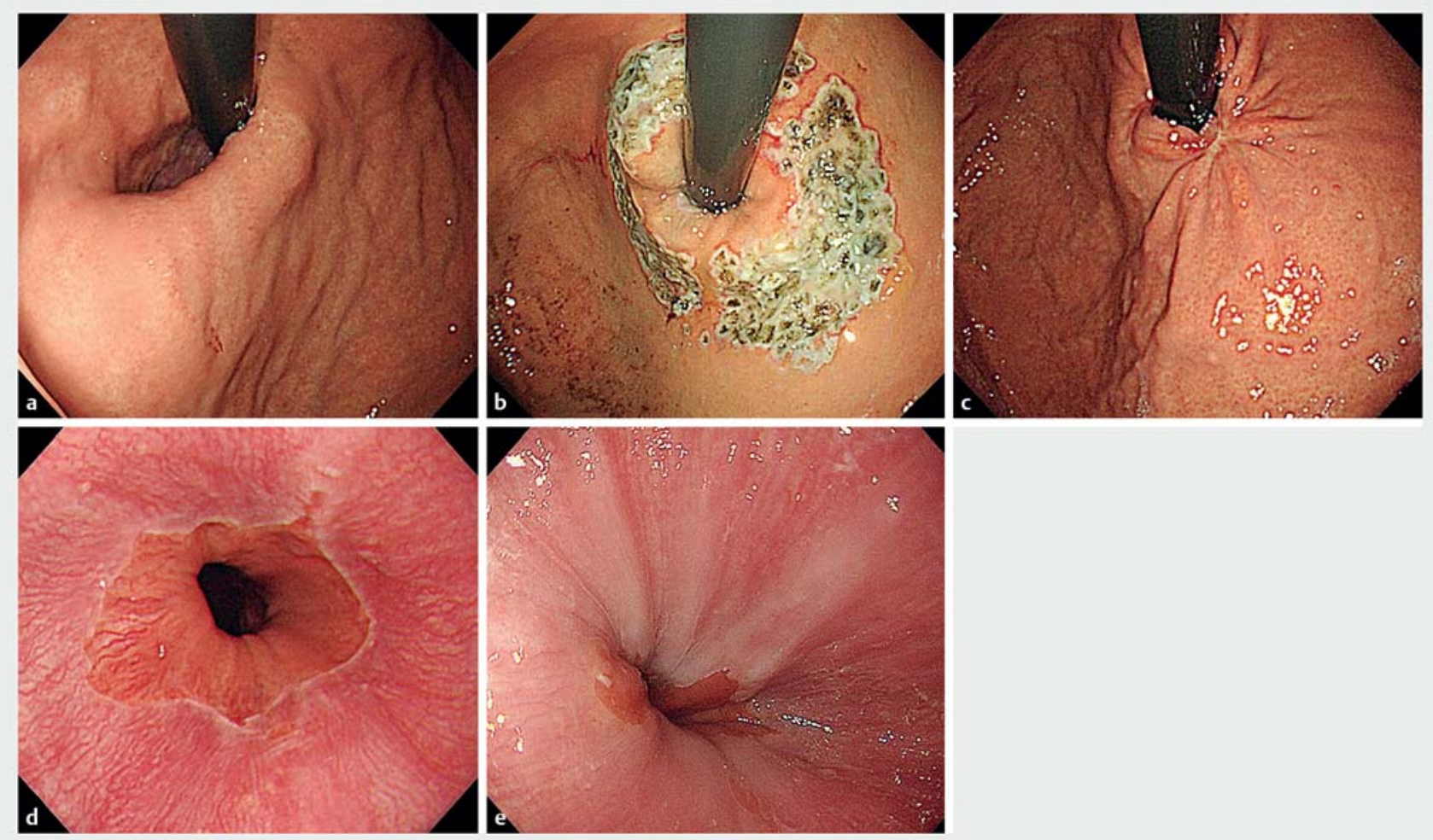

- Fig. 2 Endoscopic follow-up of anti-reflux mucosal ablation (ARMA). a Pre-ARMA. Endoscopy in retroflexion demonstrated significant hernia (Flap valve grade III) but no sliding component. b Immediately post-ARMA. Endoscopy in retroflexion showed butterfly-shaped artificial ulcer. c Appearance at 1 month post-ARMA. Mucosal flap valve was re-shaped (Flap valve grade I). $\mathbf{d}$ Before ARMA. Los Angeles grade A esophagitis is seen. e After ARMA. Erosive esophagitis resolved.

\section{Results}

Twelve consecutive patients with PPI-refractory GERD underwent ARMA (median age, 54.5 years; range, 29-75). Baseline characteristics are summarized in $>$ Table 1 . Two patients had previously undergone ARMS.

Perioperative results are shown in $\triangleright$ Table 2 . In all cases, mucosal ablation of the scheduled area was completed. The median GERD-HRQL score improved significantly from 30.5 (range, 16-45) pre-ARMA to 12 (range, $0-27)$ post-ARMA $(P=0.002)$ ( $\triangleright$ Fig.3a). The median FSSG score improved significantly from 25 (range, 13-39) pre-ARMA to 10.5 (range, 1-31) post-ARMA $(P=0.002)$ ( $\triangleright$ Fig. 3b). The median Hill's flap valve grade also exhibited a significant improvement from 1.9 to $0.5(P=0.002)$.

Ten out of 12 patients were evaluated using MII-pH monitoring at 2 months post-ARMA; two patients refused to undergo another MII-pH procedure. Moreover, two patients were excluded as they had previously undergone ARMS.Thus, final MII-pH monitoring was analyzed for eight patients. The median DeMeester score was significantly reduced from 33.5 to $2.8(P=$ 0.049 ) ( Fig.4a). The median AET showed a decreasing trend from $9 \%$ to $0.5 \%(P=0.068)$ ( $\nabla$ Fig. $4 \mathbf{b})$. Five out of eight patients showed a normalized DeMeester score $(<14.7)$ postARMA, allowing PPI discontinuation. The remaining three patients were still on PPI; however, their symptom scores improved significantly.
There were no immediate adverse events such as bleeding or perforation. One patient developed dysphagia 2 weeks postARMA due to stricture at the esophagogastric junction. The stricture was successfully managed by two sessions of balloon dilatations (with balloon diameter $13.5 \mathrm{~mm}$ ) and the patient is now free from symptoms related to stenosis. Median hospital length of stay was 4 days (range, $0-7$ ). One patient underwent ARMA under conscious propofol-based sedation and was discharged on the day of the procedure.

\section{Discussion}

The current prospective, interventional trial comprising patients with PPI-refractory GERD undergoing ARMA revealed that ARMA was well-tolerated and improved GERD-related symptoms and acid reflux by narrowing the cardiac opening, resulting in gastroesophageal reflux suppression.

Esophageal reflux is normally prevented by the anti-reflux barrier, which is a complex anatomic zone comprising the lower esophageal sphincter, extrinsic crural diaphragm and supporting structures of the gastroesophageal flap valve [12]. All patients in the current study had grade II or III Hill's flap valve, which was considered to be the primary cause of refractory GERD $[11,13]$. 
- Table 1 Baseline characteristics for patients in this study $(n=12)$.

\begin{tabular}{|c|c|}
\hline Age, median (range), years & $54.5(29-75)$ \\
\hline \multicolumn{2}{|l|}{ Sex } \\
\hline - Female, n (\%) & $5(42 \%)$ \\
\hline - Male, n (\%) & $7(58 \%)$ \\
\hline Duration of GERD symptoms, median (range), years & $6.7(1-20)$ \\
\hline \multicolumn{2}{|l|}{ Esophagitis (LA classification) } \\
\hline - None, n (\%) & $9(75 \%)$ \\
\hline - Grade A, n (\%) & $2(17 \%)$ \\
\hline - Grade B, n (\%) & $1(8 \%)$ \\
\hline \multicolumn{2}{|l|}{ Hill's flap valve grade } \\
\hline - Grade II, n (\%) & $1(8 \%)$ \\
\hline - Grade III, n (\%) & $11(92 \%)$ \\
\hline \multicolumn{2}{|l|}{ Major symptoms } \\
\hline - Heartburn, n (\%) & $8(67 \%)$ \\
\hline - Regurgitation, n (\%) & $6(50 \%)$ \\
\hline - Respiratory symptoms, n (\%) & $1(8 \%)$ \\
\hline - Chest pain, $n(\%)$ & $1(8 \%)$ \\
\hline - Belching, n (\%) & $1(8 \%)$ \\
\hline \multicolumn{2}{|l|}{ Previous intervention } \\
\hline - None, n (\%) & $10(83 \%)$ \\
\hline - ARMS, n (\%) & $2(17 \%)$ \\
\hline
\end{tabular}

- Table 2 Perioperative results for patients in this study $(n=12)$.

\begin{tabular}{|l|l|}
\hline Technical success, $\mathbf{n}(\%)$ & $12 / 12(100 \%)$ \\
\hline Total operation time, median (range), minutes & $40.3(20-69)$ \\
\hline Adverse events, $\mathbf{n}(\%)$ & \\
\hline - Bleeding, perforation & $0(0 \%)$ \\
\hline - Mild stenosis & $1(8.3 \%)$ \\
\hline Postoperative stay, median (range), days & $4(0-7)$ \\
\hline
\end{tabular}

Although LARS is considered to be the gold standard for PPIrefractory GERD, side effects including dysphagia, gas-bloat syndrome or inability to vomit are not uncommon [5]. Therefore, minimally invasive alternatives are needed. Various endoluminal techniques have been developed including transoral incisionless fundoplication [14] and radiofrequency energy delivery [15]; however, no procedure has been accepted as the standard endoscopic treatment, especially in Japan, owing to costly proprietary device requirements. ARMA does not require specific expensive devices and does not leave any artificial material in situ.

Another important strength of ARMA is that it can be repeated regardless of the presence of fibrosis from previous therapies. The first ARMA ( $\triangleright$ Fig. 1) was conducted in a patient who previously underwent ARMS. ARMA was performed due to the patient's hesitation to undergo LARS and the technical and safety issues related to a second ARMS. ARMA improved the flap valve grade and ultimately resolved the patient's symptoms. Our experience with the patient suggested that mucosal resection can be replaced by ablation with a similar effect and
GERD-HRQL

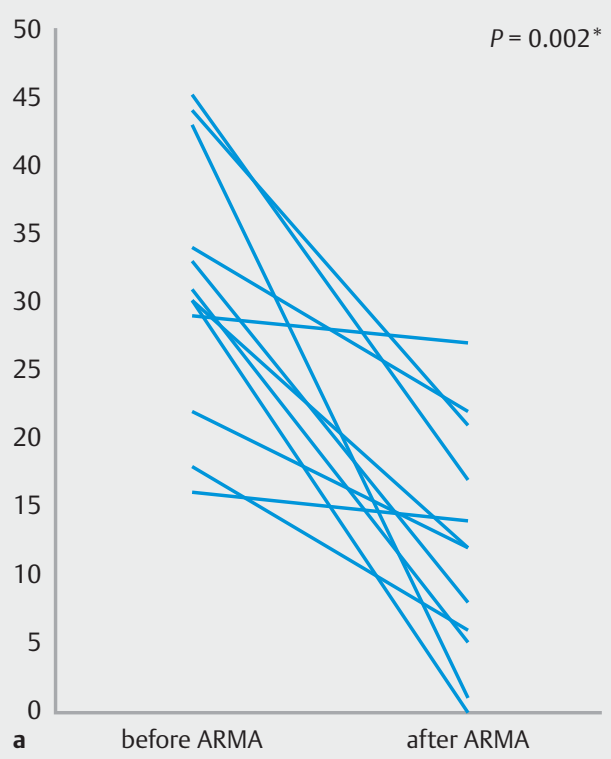

F-scale

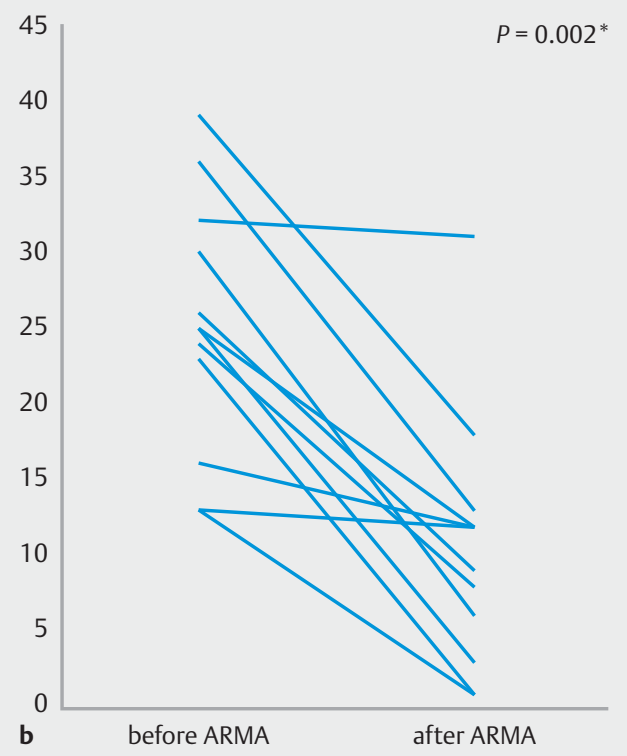

Fig. 3 Symptom scores pre- and post-ARMA ( $\mathrm{n}=12)$. a GERD-health-related quality of life score (GERD-HRQL) pre- and 2 months post-ARMA. b Frequency scale for the symptoms of gastroesophageal reflux disease (FSSG) pre- and 2 months post-ARMA. *Wilcoxon matched-pairs signed-ranks test. 

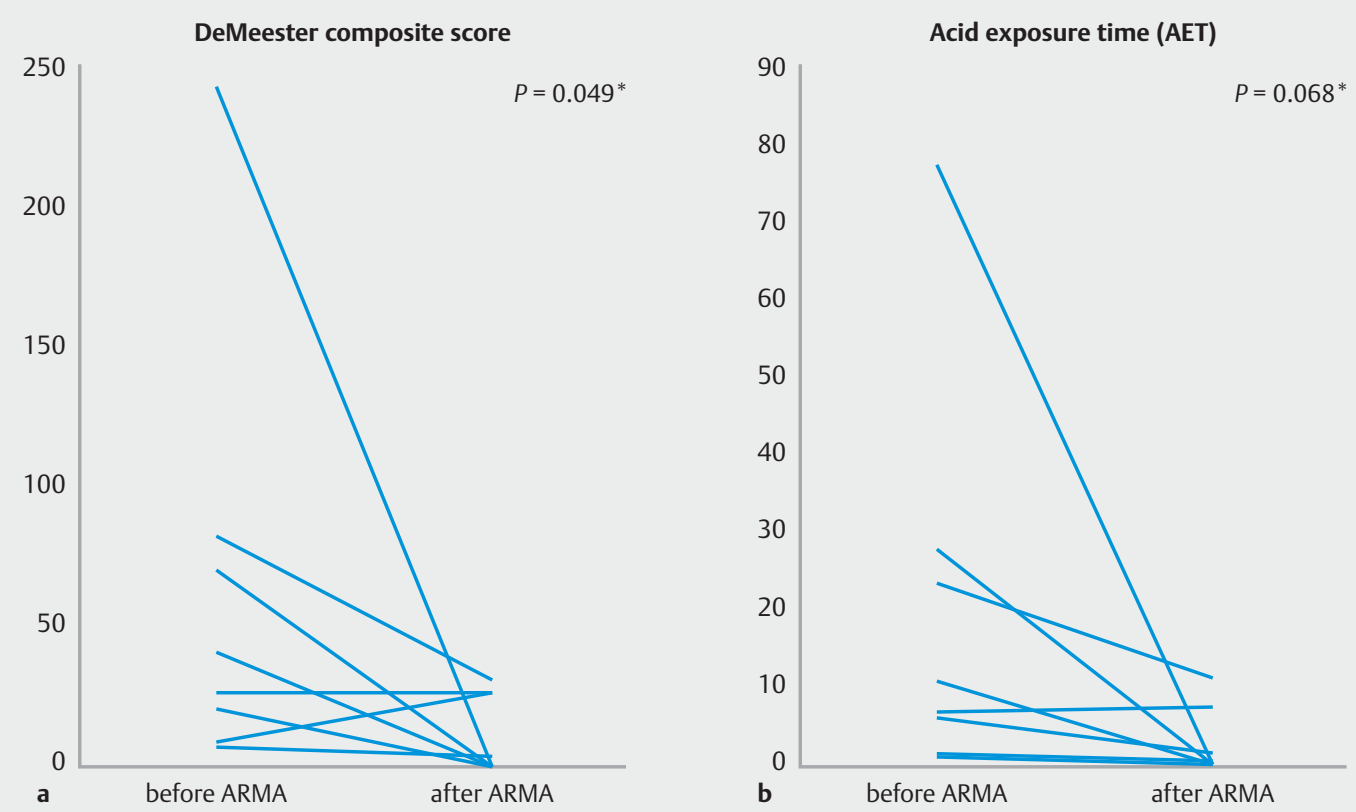

- Fig. 4 MII-pH monitoring data pre- and post-ARMA $(n=8)$. a Median DeMeester score pre- and post-ARMA significantly improved from 33.5 to $2.8(P=0.049)$. b Median Acid Exposure Time $(\mathrm{pH}<4)$ decreased from $9.0 \%$ to $0.5 \%(P=0.068)$. ${ }^{*}$ Wilcoxon matched-pairs signed-ranks test.

the advantage of repeatability. In contrast to EMR, the ablation range and depth can be controlled, and the risk of perforation seems very low as ARMA does not require resection. To achieve an effect equivalent to ARMS, ablation should be deep enough to reach the submucosal space; therefore, submucosal injection is required for safety reasons.

Ideal candidates for ARMA are patients with PPI-refractory GERD with mild to moderate gastroesophageal junction morphology impairment (hiatus hernia $<3 \mathrm{~cm}$ and maximum Hill's flap valve grade $\leq$ III). It is unlikely that ARMA will completely replace LARS as ARMA may only be effective in the absence of a significant sliding hernia. We hypothesize that another optimal indication could be GERD after gastrectomy including sleeve gastrectomy, which is not an appropriate indication for LARS. ARMA in PPI-dependent patients merits evaluation as this growing patient population may also benefit from the technique.

At this point, certain study limitations must be acknowledged. Since this was a pilot, single-arm study, only a small number of patients with outcomes of short follow-up were assessed. Randomized trials are necessary to compare the outcomes of patients treated with ARMA and other methods including ARMS. The assessment by an unblinded single operator, and the lack of $\mathrm{pH}$ monitoring data in some patients are also limitations. A single experienced operator performed all procedures so the learning curve may be a potential limiting factor as well. However, we believe that ARMA is easier than ESD or capassisted EMR.

In conclusion, ARMA is a new endoscopic method for PPI-refractory GERD. Our pilot study suggests that ARMA is simple, safe, and improves GERD-related symptoms and objective acid reflux parameters. A larger study with long-term follow-up is required to confirm these results and to evaluate predictors of treatment success to optimize patient selection.

\section{Competing interests}

Dr. Inoue is an advisor for Olympus Corporation and Top Corporation. He has also received educational grants from Olympus Corp. and Takeda Pharmaceutical Co.

\section{References}

[1] Bredenoord AJ, Pandolfino JE, Smout AJ. Gastro-oesophageal reflux disease. Lancet 2013; 381: 1933-1942

[2] Herbella FA, Patti MG. Gastroesophageal reflux disease: From pathophysiology to treatment. World J Gastroenterol 2010; 16: 3745-3749

[3] Dean BB, Gano AD Jr., Knight K et al. Effectiveness of proton pump inhibitors in nonerosive reflux disease. Clin Gastroenterol Hepatol 2004; 2: 656-664

[4] Vaezi MF, Yang YX, Howden CW. Complications of proton pump inhibitor therapy. Gastroenterology 2017; 153: 35-48

[5] Pandolfino JE, Krishnan K. Do endoscopic antireflux procedures fit in the current treatment paradigm of gastroesophageal reflux disease? Clin Gastroenterol Hepatol 2014; 12: 544-554

[6] Yadlapati R, Vaezi MF, Vela MF et al. Management options for patients with GERD and persistent symptoms on proton pump inhibitors: recommendations from an expert panel. Am J Gastroenterol 2018; 113: 980-986

[7] Hopkins ], Switzer N], Karmali S. Update on novel endoscopic therapies to treat gastroesophageal reflux disease: A review. World J Gastrointest Endosc 2015; 7: 1039-1044 
[8] Inoue $\mathrm{H}$, Ito $\mathrm{H}$, Ikeda $\mathrm{H}$ et al. Anti-reflux mucosectomy for gastroesophageal reflux disease in the absence of hiatus hernia: a pilot study. Ann Gastroenterol 2014; 27: 346-351

[9] Velanovich V. The development of the GERD-HRQL symptom severity instrument. Dis Esophagus 2007; 20: 130-134

[10] Kusano M, Shimoyama Y, Sugimoto $S$ et al. Development and evaluation of FSSG: frequency scale for the symptoms of GERD. J Gastroenterol 2004; 39: 888-891

[11] Hill LD, Kozarek RA, Kraemer S] et al. The gastroesophageal flap valve: in vitro and in vivo observations. Gastrointest Endosc 1996; 44: $541-$ 547
[12] Tack J, Pandolfino JE. Pathophysiology of gastroesophageal reflux disease. Gastroenterology 2018; 154: 277-288

[13] van Herwaarden MA, Samsom M, Smout AJ. The role of hiatus hernia in gastro-oesophageal reflux disease. Eur J Gastroenterol Hepatol 2004; 16: 831-835

[14] Testoni PA, Testoni S, Mazzoleni G et al. Long-term efficacy of transoral incisionless fundoplication with Esophyx (Tif 2.0) and factors affecting outcomes in GERD patients followed for up to 6 years: a prospective single-center study. Surg Endosc 2015; 29: 2770-2780

[15] Noar M, Squires P, Noar E et al. Long-term maintenance effect of radiofrequency energy delivery for refractory GERD: a decade later. Surg Endosc 2014; 28: 2323-2333 